PROCEEDINGS OF THE

AMERICAN MATHEMATICAL SOCIETY

Volume 129, Number 2, Pages 601-607

S 0002-9939(00)05665-3

Article electronically published on August 29, 2000

\title{
TYPES IN REDUCTIVE $p$-ADIC GROUPS: THE HECKE ALGEBRA OF A COVER
}

\author{
COLIN J. BUSHNELL AND PHILIP C. KUTZKO \\ (Communicated by Rebecca A. Herb)
}

\begin{abstract}
In this paper, $F$ is a non-Archimedean local field and $G$ is the group of $F$-points of a connected reductive algebraic group defined over $F$. Also, $\tau$ is an irreducible representation of a compact open subgroup $J$ of $G$, the pair $(J, \tau)$ being a type in $G$. The pair $(J, \tau)$ is assumed to be a cover of a type $\left(J_{L}, \tau_{L}\right)$ in a Levi subgroup $L$ of $G$. We give conditions, generalizing those of earlier work, under which the Hecke algebra $\mathcal{H}(G, \tau)$ is the tensor product of a canonical image of $\mathcal{H}\left(L, \tau_{L}\right)$ and a sub-algebra $\mathcal{H}(K, \tau)$, for a compact open subgroup $K$ of $G$ containing $J$.
\end{abstract}

We return to our earlier paper 2 in order to simplify and generalize one of its more suggestive results.

1.1. Let $F$ be a non-Archimedean local field and $G$ the group of $F$-rational points of a connected reductive algebraic group defined over $F$. We write $\mathfrak{R}(G)$ for the category of all smooth representations of $G$. As in [2], $\S 1$, we form the set $\mathcal{B}(G)$ : this consists of $G$-inertial equivalence classes of pairs $(L, \sigma)$, where $L$ is an $F$-Levi subgroup of $G$ and $\sigma$ is an irreducible supercuspidal representation of $L$. As in [2], 1.4 , and [1], 2.10, this leads to a canonical decomposition

$$
\mathfrak{R}(G)=\prod_{\mathfrak{s} \in \mathcal{B}(G)} \mathfrak{R}^{\mathfrak{s}}(G)
$$

of $\mathfrak{R}(G)$ as a direct product of certain full sub-categories $\mathfrak{R}^{\mathfrak{s}}(G)$.

1.2. In [2], we considered the problem of describing the factor categories $\mathfrak{R}^{\mathfrak{s}}(G)$ via certain representations of compact open subgroups of $G$, which we call types. Recall that an irreducible smooth representation $(\rho, U)$ of a compact open subgroup $K$ of $G$ is an $\mathfrak{s}$-type if those irreducible representations of $G$ which contain $\rho$ are precisely those which lie in $\mathfrak{R}^{\mathfrak{s}}(G)$. In these circumstances, the category $\mathfrak{R}^{\mathfrak{s}}(G)$ is canonically equivalent to the category of (left) modules over the convolution algebra $\mathcal{H}(G, \rho)$ of compactly supported $\rho$-spherical functions on $G$. More precisely, if $(\pi, V) \in \mathfrak{R}(G)$, the space $V_{\rho}=\operatorname{Hom}_{K}(U, V)$ is a module over $\mathcal{H}(G, \rho)$; the equivalence $\mathfrak{R}^{\mathfrak{s}}(G) \rightarrow \mathcal{H}(G, \rho)$-Mod is then given by $(\pi, V) \mapsto V_{\rho}$. It will therefore be useful to construct types $(K, \rho)$ and describe the associated algebras $\mathcal{H}(G, \rho)$.

Received by the editors April 28, 1999.

1991 Mathematics Subject Classification. Primary 22E50, 22D99.

Key words and phrases. p-adic reductive group, type, cover, Hecke algebra.

The research of the second-named author was partially supported by NSF grant DMS-9003213. 
1.3. The method of covers, described in $2, \S 8$, gives an axiomatic approach to this problem. Suppose that $\mathfrak{s} \in \mathcal{B}(G)$ is the $G$-inertial equivalence class of a pair $(L, \sigma)$. This pair also defines an element $\mathfrak{t} \in \mathcal{B}(L)$. For the purposes of this paper, we assume that the supercuspidal representation $\sigma$ has certain auxiliary properties as follows:

Assumptions. (i) There are an open compact mod centre subgroup $\widetilde{J}_{L}$ of $L$ and an irreducible smooth representation $\widetilde{\tau}_{L}$ of $\widetilde{J}_{L}$ such that $\sigma \cong c$-Ind $\widetilde{\tau}_{L}$.

(ii) The representation $\tau_{L}=\widetilde{\tau}_{L} \mid J_{L}$ is irreducible, where $J_{L}$ denotes the unique maximal compact subgroup of $\widetilde{J}_{L}$.

(iii) An element $x \in L$ intertwines $\tau_{L}$ if and only if $x \in \widetilde{J}_{L}$.

We note that these are precisely the conditions 2, 5.5. They are satisfied, in particular, if $\sigma$ is "of level zero" [3] or if $L$ is essentially a product of general linear groups.

The pair $\left(J_{L}, \tau_{L}\right)$ is a $\mathfrak{t}$-type in $L$; the Hecke algebra $\mathcal{H}\left(L, \tau_{L}\right)$ is commutative and canonically identified with the centre of the category $\mathfrak{R}^{\mathfrak{t}}(L)$. In particular, it is the ring of regular functions on a certain complex algebraic torus. (For these assertions, see [2], §5.)

1.4. In the same situation, we assume given a $G$-cover $(J, \tau)$ of $\left(J_{L}, \tau_{L}\right)$. There is no need to recall the definition, only that $(J, \tau)$ is an $\mathfrak{s}$-type in $G[2], 8.3$, and that, given a parabolic subgroup $P$ of $G$ with Levi component $L$, there is a canonical algebra homomorphism $t_{P}: \mathcal{H}\left(L, \tau_{L}\right) \rightarrow \mathcal{H}(G, \tau)$, as in [2], 7.12. This homomorphism is injective, so we can identify $\mathcal{H}\left(L, \tau_{L}\right)$ with a subalgebra $\mathcal{B}_{P}$ of $\mathcal{A}=\mathcal{H}(G, \tau)$.

1.5. The $G$-normalizer $\mathrm{N}_{G}(L)$ acts on the set $\mathcal{B}(L)$ in a natural way, each point being fixed by $L$. We write $\mathbf{W}_{\mathfrak{s}}$ for the (finite) $\mathrm{N}_{G}(L) / L$-stabilizer of $\mathfrak{t}$.

Now let $K$ be some compact subgroup of $G$ which contains $J$. We can then form the sub-algebra $\mathcal{K}=\mathcal{H}(K, \tau)$ of $\mathcal{A}$ consisting of functions with support contained in $K$. Using $\star$ to denote convolution in $\mathcal{A}$, our main result is:

Theorem. (i) The map

$$
\begin{gathered}
\mathcal{B}_{P} \otimes_{\mathbb{C}} \mathcal{K} \longrightarrow \mathcal{A}, \\
f \otimes \phi \longmapsto f \star \phi
\end{gathered}
$$

is an injective homomorphism of $\left(\mathcal{B}_{P}, \mathcal{K}\right)$-bimodules.

(ii) We have $\operatorname{dim}_{\mathbb{C}}(\mathcal{K}) \leqslant\left|\mathbf{W}_{\mathfrak{s}}\right|$.

(iii) If $\operatorname{dim}_{\mathbb{C}}(\mathcal{K})=\left|\mathbf{W}_{\mathfrak{s}}\right|$, then the map of (i) is an isomorphism.

1.6. In [2, 11.4, we obtained a similar result involving several hypotheses on the group $K$. Prompted by his results in [3], Lawrence Morris queried whether these restrictions were really necessary. The present paper is a response to his comments.

Many of the arguments required to prove Theorem 1.5 parallel those of [2], so we do not repeat them. However, the technical preparation is rather different. It is this which provides the bulk of the paper, and is the source of such novelty as it possesses.

\section{Compact induction and Jacquet functors}

2.1. For this section only, we let $G$ be a locally profinite (i.e. locally compact and totally disconnected) topological group. We write $\mathfrak{R}(G)$ for the category of smooth (complex) representations of $G$. 
Let $H$ be a locally profinite group, and let $\phi: G \rightarrow H$ be a continuous homomorphism. We thus have the "restriction" functor $\phi^{*}: \mathfrak{R}(H) \rightarrow \mathfrak{R}(G)$. Explicitly, if $(\sigma, V) \in \mathfrak{R}(H)$, then $\phi^{*}(\sigma, V)=\left(\phi^{*} \sigma, V\right)$, where $\phi^{*} \sigma(g)=\sigma(\phi(g)), g \in G$.

2.2. We are now given a locally profinite group $P$ together with a closed normal subgroup $N$ of $P$. We put $M=P / N$ and write $\pi: P \rightarrow M$ for the quotient map. We fix a compact open subgroup $K$ of $P$, and write $\iota: K \rightarrow P$ for the inclusion map. The functor $\pi^{*}: \mathfrak{R}(M) \rightarrow \mathfrak{R}(P)$ is then inflation of representations, while $\iota^{*}: \mathfrak{R}(P) \rightarrow \mathfrak{R}(K)$ is the ordinary restriction of representations.

2.3. Each of the functors $\pi^{*}, \iota^{*}$ admits a co-adjoint. The co-adjoint of $\pi^{*}$ is the Jacquet functor $\pi_{*}:(\sigma, V) \mapsto\left(\sigma_{N}, V_{N}\right)$. Here, $V_{N}=V / V(N)$, where $V(N)$ is the subspace of $V$ spanned by the set

$$
\{v-\sigma(n) v: v \in V, n \in N\}
$$

The action of $M$ is then given by

$$
\sigma_{N}(\pi(p)): v+V(N) \longmapsto \sigma(p) v+V(N), \quad p \in P .
$$

We write $r_{N}=r_{N}(V)$ for the quotient map $V \rightarrow V_{N}$.

2.4. On the other hand, a co-adjoint $\iota_{*}$ for $\iota^{*}$ is given by the functor $c$ - $\operatorname{Ind}_{K}^{P}$ of compact induction (see, for example, [2], §2). Explicitly, for $(\rho, U) \in \mathfrak{R}(K)$, the representation $\iota_{*}(\rho, U)=\left(\iota_{*} \rho, \iota_{*} U\right)$ is given as follows. The space $\iota_{*} U$ consists of all compactly supported functions $f: G \rightarrow U$ such that $f(k g)=\rho(k) f(g), k \in K$, $g \in G$. For $g \in G$, the function $\iota_{*} \rho(g) f: x \mapsto f(x g)$. In particular, we have a canonical $K$-injection

$$
\begin{gathered}
U \longrightarrow \iota_{*} U, \\
u \longmapsto f_{u},
\end{gathered}
$$

where $f_{u}$ has support $K$ and $f_{u}(1)=u$. The co-adjoint isomorphism

$$
\begin{array}{ll}
\operatorname{Hom}_{P}(c-\operatorname{Ind}(U), V) \stackrel{\approx}{\longrightarrow} \operatorname{Hom}_{K}(U, V), \quad V \in \mathfrak{R}(K), \\
V \in \mathfrak{R}(P),
\end{array}
$$

is then given by composition with the map $u \mapsto f_{u}$.

2.5. We now define $\phi: K \rightarrow M$ by $\phi=\pi \circ \iota$. Then clearly $\phi^{*}=\iota^{*} \circ \pi^{*}$, whence $\pi_{*} \circ \iota_{*}$ is a co-adjoint for $\phi^{*}$.

On the other hand, the map $\phi$ factors as $\phi=\iota^{M} \circ \pi^{K}$, where $\pi^{K}: K \rightarrow$ $K /(K \cap N)$ is the canonical surjection and $\iota^{M}: K /(K \cap N) \rightarrow M$ is the canonical injection. Just as in 2.3, the functor $\left(\iota^{M}\right)^{*}$ admits the co-adjoint $\iota_{*}^{M}=c$-Ind $K_{M}^{M}$, where we abbreviate $K_{M}=K / K \cap N$. Likewise, $\left(\pi^{K}\right)^{*}$ has co-adjoint

$$
\pi_{*}^{K}:(\rho, U) \longmapsto\left(\rho_{K \cap N}, U_{K \cap N}\right) .
$$

Thus $\iota_{*}^{M} \circ \pi_{*}^{K}$ is also co-adjoint to $\phi^{*}$. By uniqueness of co-adjoint functors, we have an isomorphism of functors

$$
\pi_{*} \circ \iota_{*} \approx \iota_{*}^{M} \circ \pi_{*}^{K} .
$$

In particular, we have a canonical $M$-isomorphism

$$
\Phi(U): c-\operatorname{Ind}_{K}^{P}(U)_{N} \stackrel{\approx}{\longrightarrow}-\operatorname{Ind}_{K_{M}}^{M}\left(U_{K \cap N}\right),
$$

for each $(\rho, U) \in \mathfrak{R}(K)$. 
Proposition. (i) The isomorphism $\Phi(U)$ of (2.5.2) is the unique $M$-map such that the following diagram commutes:

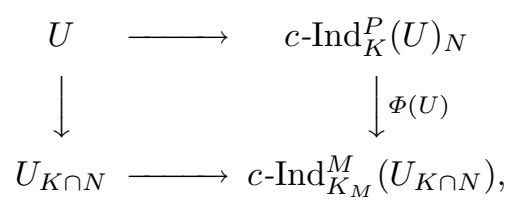

where the top row is the composite of canonical maps

$$
U \stackrel{u \mapsto f_{u}}{\longrightarrow} c-\operatorname{Ind}(U) \stackrel{r_{N}}{\longrightarrow} c-\operatorname{Ind}(U)_{N} .
$$

(ii) Let $\mathcal{C}$ be the space of functions in $c-\operatorname{Ind}(U)$ with support contained in $K$, and $\mathcal{C}_{M}$ the space of functions in $c-\operatorname{Ind}\left(U_{K \cap N}\right)$ with support contained in $K_{M}$. Then $\Phi(U)$ induces an isomorphism $r_{N}(\mathcal{C}) \cong \mathcal{C}_{M}$.

Proof. Part (i) follows easily from the discussion in 2.4. In part (ii), the space $\mathcal{C}$ is the image of the canonical map $U \rightarrow c$-Ind $(U)$, and similarly for $\mathcal{C}_{M}$. The assertion now follows from (i).

\section{Ideals of Hecke Algebras and COMPaCt subgroups}

In this section, we prove the central technical result of the paper. This generalizes a lemma from [2], $\S 11$.

3.1. We revert to the notation and assumptions of $\S 1$. As before, we set $\mathcal{A}=$ $\mathcal{H}(G, \tau)$, and we abbreviate $\mathcal{H}_{L}=\mathcal{H}\left(L, \tau_{L}\right)$. We write $U$ for the representation space of $\tau$ and $\tau_{L}$.

Given a smooth representation $(\pi, V)$ of $G$, we recall that the space $V_{\tau}$ of $\tau$ invariants in $V$, defined by $V_{\tau}=\operatorname{Hom}_{K}(U, V)$, is a left $\mathcal{A}$-module, in a natural way $([2, \S 2)$.

3.2. We are given a compact open subgroup $K$ of $G$ containing $J$, and a finitedimensional smooth representation $(\rho, W)$ of $K$. We set

$$
(\omega, \mathcal{X})=c-\operatorname{Ind}_{K}^{G}(\rho, W) .
$$

We let $\mathcal{C}$ denote the space of functions in $\mathcal{X}$ with support contained in $K$. Thus $\mathcal{C}$ is a $K$-subspace of $\mathcal{X}$, and we view $\mathcal{C}_{\tau}=\operatorname{Hom}_{K}(U, \mathcal{X})$ as a subspace of $\mathcal{X}_{\tau}$.

Finally, we fix a parabolic subgroup $P$ of $G$ with Levi component $L$. As in [2], 7.12, this gives us an injective algebra homomorphism $t_{P}: \mathcal{H}_{L} \rightarrow \mathcal{A}$. We write $\mathcal{B}_{P}=t_{P}\left(\mathcal{H}_{L}\right)$, and we view $\mathcal{X}_{\tau}$ as an $\mathcal{B}_{P}$-module. When convenient, we view it as $\mathcal{H}_{L}$-module via the isomorphism $t_{P}: \mathcal{H}_{L} \cong \mathcal{B}_{P}$.

We now prove:

Proposition. Let $\mathcal{I}$ be a maximal ideal of the algebra $\mathcal{B}_{P}$. Then

$$
\mathcal{I} \mathcal{X}_{\tau} \cap \mathcal{C}_{\tau}=\{0\}
$$

3.3. The proof of Proposition 3.2 starts with a lemma:

Lemma. (i) For $g \in G$, let $\mathcal{X}[g]$ denote the set of functions in $\mathcal{X}$ with support contained in $\mathrm{KgP}$. Then $\mathcal{X}[g]$ is a $P$-subspace of $\mathcal{X}$ and we have

$$
\mathcal{X}=\bigoplus_{g \in K \backslash G / P} \mathcal{X}[g] .
$$


(ii) Let $f \in \mathcal{X}[g]$, and define a function $f^{P}: P \rightarrow U$ by $f^{P}(p)=f(g p), p \in P$. Then $f \mapsto f^{P}$ induces a $P$-isomorphism

$$
\mathcal{X}[g] \cong c-\operatorname{Ind}_{g^{-1} K g \cap P}^{P}\left(\rho^{g}\right) .
$$

Proof. Straightforward.

We remark that, in (ii), $\rho^{g}$ denotes as usual the representation $x \mapsto \rho\left(g x g^{-1}\right)$ of the group $K^{g}=g^{-1} K g$.

3.4. We now prove Proposition 3.2. We write $r_{N}: \mathcal{X} \rightarrow \mathcal{X}_{N}$ for the canonical quotient map. Composition with $r_{N}$ then induces a map $q: \mathcal{X}_{\tau} \rightarrow\left(\mathcal{X}_{N}\right)_{\tau_{L}}$, which is an isomorphism of $\mathcal{H}_{L}$-modules ([2], Theorem 7.9). Also, $q\left(\mathcal{C}_{\tau}\right) \subset r_{N}(\mathcal{C})_{\tau_{L}}$. It is therefore enough to show that

$$
\mathcal{I}\left(\mathcal{X}_{N}\right)_{\tau_{L}} \cap r_{N}(\mathcal{C})_{\tau_{L}}=\{0\}
$$

for any maximal ideal $\mathcal{I}$ of $\mathcal{H}_{L}$.

Consider the space $\mathcal{X}[1]=\mathcal{X}\left[1_{G}\right]$, in the notation of Lemma 3.3. We have $\mathcal{C} \subset \mathcal{X}[1]$, so $r_{N}(\mathcal{C}) \subset \mathcal{X}[1]_{N}$. On the other hand, Lemma 3.3 implies

$$
\left(\mathcal{X}_{N}\right)_{\tau_{L}}=\bigoplus_{g \in K \backslash G / P}\left(\mathcal{X}[g]_{N}\right)_{\tau_{L}}
$$

so we have to show that

$$
\mathcal{I}\left(\mathcal{X}[1]_{N}\right)_{\tau_{L}} \cap r_{N}(\mathcal{C})_{\tau_{L}}=\{0\}
$$

for any maximal ideal $\mathcal{I}$ of $\mathcal{H}_{L}$.

The $P$-isomorphism $\mathcal{X}[1] \rightarrow c$ - $\operatorname{Ind}_{K \cap P}^{P}(\rho)$ given by Lemma 3.3 carries $\mathcal{C}$ isomorphically to the space $\mathcal{C}_{P}$ of functions in $c$-Ind $\operatorname{In\cap P}_{K}^{P}(\rho)$ with support in $K \cap P$. On the other hand, Proposition 2.5 gives us an $L$-isomorphism

$$
\mathcal{X}[1]_{N} \cong c-\operatorname{Ind}_{K_{L}}^{L}\left(U_{K \cap N}\right),
$$

where $K_{L}=K \cap P / K \cap N$. Taken together, these maps give us an isomorphism

$$
\left(\mathcal{X}[1]_{N}\right)_{\tau_{L}} \cong c-\operatorname{Ind}_{K_{L}}^{L}\left(U_{K \cap N}\right)_{\tau_{L}}
$$

of $\mathcal{H}_{L}$-modules which takes $r_{N}(\mathcal{C})_{\tau_{L}}$ to $\left(\mathcal{C}_{L}\right)_{\tau_{L}}$, where $\mathcal{C}_{L}$ is the space of functions in $c$ - $\operatorname{Ind}_{K_{L}}^{L}\left(U_{K \cap N}\right)$ with support in $K_{L}$. The result now follows from [2], 5.10.

3.5. We now give a consequence of Proposition 3.2.

Corollary. Let $\mathcal{K}$ denote the sub-algebra of functions in $\mathcal{A}$ with support contained in $K$. The canonical map $\mathcal{K} \rightarrow \mathcal{A} /(\mathcal{I} \star \mathcal{H})$ is then injective for any maximal ideal $\mathcal{I}$ of $\mathcal{B}_{P}$.

Proof. We apply the proposition to the representation $(\rho, U)=c-\operatorname{Ind}_{J}^{K}(\tau)$. Thus $(\omega, \mathcal{X})=c$-Ind $\operatorname{In}_{J}^{G}(\tau)$. We thus have an $\mathcal{A}$-isomorphism $\mathcal{A} \cong \mathcal{X}_{\tau}$ under which $\mathcal{K}$ gets mapped to $\mathcal{C}_{\tau}$, whence the result.

\section{The MAIN THEOREM}

We now prove Theorem 1.5.

4.1. We continue with the notation of $\S \S 1,3$. In this section, we are given a compact open subgroup $K$ of $G$ containing $J$.

We write $\mathcal{K}=\mathcal{H}(K, \tau)$ for the algebra of functions in $\mathcal{A}$ with support contained in $K$. 
4.2. Since the parabolic subgroup $P$ will be fixed throughout, we abbreviate $\mathcal{B}=$ $\mathcal{B}_{P}$.

Let $\mathcal{I}$ be a maximal ideal of $\mathcal{B}$. We observe that the space $\mathcal{A} / \mathcal{I} \star \mathcal{A}$ has complex dimension $\left|\mathbf{W}_{\mathfrak{s}}\right|$ : the argument in the proof of [2] 11.5(ii) is independent of $K$ and so applies here. By Corollary 3.5 , we get $\operatorname{dim}_{\mathbb{C}} \mathcal{K} \leqslant\left|\mathbf{W}_{\mathfrak{s}}\right|$, which is part (ii) of the theorem.

We next prove part (i) of the theorem, that the obvious map $\mathcal{B} \otimes_{\mathbb{C}} \mathcal{K} \rightarrow \mathcal{B} \star \mathcal{K}$ is injective. Let $\mathcal{L}$ denote the kernel of this map, and put $k=\operatorname{dim}_{\mathbb{C}} \mathcal{K}$. Fix a maximal ideal $\mathcal{I}$ of $\mathcal{B}$, and consider the exact sequence

$$
0 \rightarrow \operatorname{Hom}_{\mathcal{B}}(\mathcal{B} \star \mathcal{K}, \mathcal{B} / \mathcal{I}) \rightarrow \operatorname{Hom}_{\mathcal{B}}(\mathcal{B} \otimes \mathcal{K}, \mathcal{B} / \mathcal{I}) \rightarrow \operatorname{Hom}_{\mathcal{B}}(\mathcal{L}, \mathcal{B} / \mathcal{I})
$$

Since $\mathcal{B}$ is the ring of regular functions on a complex algebraic variety, we have $\mathcal{B} / \mathcal{I}=\mathbb{C}$. The complex space $\operatorname{Hom}_{\mathcal{B}}(\mathcal{B} \otimes \mathcal{K}, \mathcal{B} / \mathcal{I})$ thus has dimension $k$. On the other hand, write $\mathcal{X}$ for the image of $\mathcal{K}$ in $\mathcal{A} / \mathcal{I} \star \mathcal{A}$; this is a $\mathbb{C}=\mathcal{B} / \mathcal{I}$ space of dimension $k$ (by Corollary 3.5), and is a $\mathcal{B}$-quotient of $\mathcal{B} \star \mathcal{K}$. We deduce that $\operatorname{Hom}_{\mathcal{B}}(\mathcal{B} \star \mathcal{K}, \mathcal{B} / \mathcal{I})$ has dimension at least $k$. It follows that the map $\operatorname{Hom}_{\mathcal{B}}(\mathcal{B} \star \mathcal{K}, \mathcal{B} / \mathcal{I}) \rightarrow \operatorname{Hom}_{\mathcal{B}}(\mathcal{B} \otimes \mathcal{K}, \mathcal{B} / \mathcal{I})$ is bijective. Now we can argue as at the end of [2] $\S 11$ to conclude $\mathcal{L}=\{0\}$, as desired. This proves part (i) of the theorem.

We now assume that $k=\left|\mathbf{W}_{\mathfrak{s}}\right|$. We then have

$$
\mathcal{A}=\mathcal{K}+\mathcal{I} \star \mathcal{A}=\mathcal{B} \star \mathcal{K}+\mathcal{I} \star \mathcal{A}
$$

for every maximal ideal $\mathcal{I}$ of $\mathcal{B}$.

From this point, we can just follow the proof of 2 Theorem 11.4, once we have established:

Lemma. $\mathcal{A}$ is finitely generated as left $\mathcal{B}$-module.

Proof. We apply the machinery of the $\S \S 2,3$ with $(\rho, U)=c-\operatorname{Ind}_{J}^{K}(\tau),(\omega, \mathcal{X})=$ $c$ - $\operatorname{Ind}_{K}^{G}(\rho) \cong c$-Ind ${ }_{J}^{G}(\tau)$. Thus $\mathcal{A} \cong \mathcal{X}_{\tau}$ as $\mathcal{A}$-module and, by [2], Theorem 7.9,

$$
\mathcal{A} \cong \mathcal{X}_{\tau} \cong\left(\mathcal{X}_{N}\right)_{\tau_{L}}
$$

as $\mathcal{B}$-module. However, by Lemma 3.3 and Proposition 2.5, we have

$$
\left(\mathcal{X}_{N}\right)_{\tau_{L}} \cong \bigoplus_{z \in K \backslash G / P} c-\operatorname{Ind}_{K_{L}^{z}}^{M}\left(U_{L}^{z}\right)_{\tau_{L}}
$$

where $K_{L}^{z}=\left(K^{z}\right)_{L}=\left(z^{-1} K z \cap P\right) /\left(z^{-1} K z \cap N\right)$ and (in the notation of of 2.5)

$$
\left(\rho_{L}^{z}, U_{L}^{z}\right)=\pi_{*}^{z^{-1} K z \cap P}\left(\rho^{z}, U^{z}\right)
$$

The set $K \backslash G / P$ is finite. Each summand $c$ - $\operatorname{Ind}_{K_{L}^{z}}^{M}\left(U_{L}^{z}\right)_{\tau_{L}}$ is finitely generated as $\mathcal{B}$-module by [2], 5.11. (We may use this result, since its proof does not require $\left.J_{L} \subset K_{L}^{z}\right)$. The lemma now follows.

Remark. The preceding lemma does not rely on any particular property of the compact subgroup $K$; indeed, we could have taken $K=J$ and $\rho=\tau$.

This completes the proof of the theorem. 


\section{REFERENCES}

1. J.-N. Bernstein (rédigé par P. Deligne), Le "centre" de Bernstein, Représentations des groupes réductifs sur un corps local, Hermann, Paris, 1984, pp. 1-32. [MR 86e:22028

2. C.J. Bushnell and P.C. Kutzko, Smooth representations of reductive p-adic groups: structure theory via types, Proc. London Math. Soc. (3) 77 (1998), 582-634. CMP 99:01

3. L.E. Morris, Tamely ramified intertwining algebras, Invent. Math. 114 (1993), 1-54. MR 94g:22035

Department of Mathematics, King's College, Strand, London WC2R 2LS, United KINGDOM

E-mail address: bushnell@mth.kcl.ac.uk

Department of Mathematics, University of Iowa, Iowa City, Iowa 52242

E-mail address: pkutzko@blue.weeg.uiowa.edu 